Pacific Journal of Mathematic 


\title{
DEFINABILITY IN THE LATTICE OF RING VARIETIES
}

\author{
AWAD A. ISKANDER
}

\begin{abstract}
A variety of associative rings $B$ immediately covers its subvariety $A$ if every member of $B$ outside $A$ generates $B$. The variety $\{2 x=0, x y=0\}$ is the unique equationally complete variety with precisely two immediate covers in the lattice of all associative ring varieties. The variety of all Boolean rings is first order definable in the lattice of all associative ring varieties. So are the varieties defined by $\{2 x=0, x y-y x=0\}$ and $\{x y=0\}$.
\end{abstract}

The set of all varieties of associative rings (not necessarily with 1 ) is a complete lattice under class inclusion. The variety of all Boolean rings is a member of this lattice. In contrast to the several equivalent axiomatizations of the theory of Boolean rings that can be found in the literature, there does not seem to exist any description of the Bloolean variety as a member of the lattice of all ring varieties. In this paper, a lattice theoretic notation is defined: $b$ immediately covers $a$ if $x \leqq a$ is equivalent to $x<b$. This notion is applied to show that the varieties defined by $\{2 x=0, x y=0\}$, $\{x y=0\},\{2 x=0, x y-y x=0\}$ and the variety of Boolean rings are all first order definable members in the lattice of all associative ring varieties.

In [5], Ralph McKenzie says that the variety of Boolean algebras is a definable member in the lattice of all varieties of universal algebras with two binary and one unary operation. The methods of [5] do not yield our results. In the present paper, the variety of Boolean rings with 1 is not defined as a member of the lattice of all varieties of associative rings with 1 , neither is the variety of Boolean rings as a member of all varieties of commutative rings.

1. It is clear that $b$ immediately covers $a$ if and only if $a<b$ and $a$ is the join of all elements of $L$ strictly below $b$. Equivalently: $b$ is an immediate cover of $a$ if and only if $b$ is completely join irreducible and $a$ is the join of all elements of $L$ that are strictly less than $b$. Thus, an element may have more than one immediate cover-the atoms of $L$ are the immediate covers of the least element 0 of $L$. On the other hand, an element can immediately cover no more than one element.

Let $A$ be a subset of $L . A$ is called first order definable in $L$ if there is a first order sentence $F(x)$ in one free variable $x$, relative 
to the lattice operations $A$ and $V$ of $L$, such that $A$ is the set of all elements $a$ of $L$ for which $F(a)$ holds. An element $a$ of $L$ is called first order definable in $L$ if the singleton $\{a\}$ is first order definable in $L$.

Thus 0 is a first order definable element in $L$. The same is true for the largest element of $L$. A set that is first order definable relative to first order definable sets is itself first order definable. The predicate " $x$ is an immediate cover of $y$ " can be expressed as a first order formula in two variables relative to meet and join. The atoms are the immediate covers of 0 and hence the set of all atoms is first order definable in $L$. The join of a definable set is a definable element in $L$.

2. In this paper of importance are varieties of associative rings. In order to fix the notations, some of the basic definitions and results are recalled here. An account of the theory can be found in $[1,2$, 6].

By the word "variety" will be meant a variety (equational class) of associative rings, that is the class of all associative rings, not necessarily with 1 , satisfying a given set of polynomial identities $\left\{P_{\alpha}\left(x_{1}, \cdots, x_{n_{\alpha}}\right)=0: \alpha \in I\right\}$. If $\mathfrak{A}_{i}, i \in M$ is a family of varieties, then their meet $\Lambda\left\{\mathfrak{A}_{i}: i \in M\right\}$ is the variety of all $A$ such that $A \in \mathfrak{A}_{i}$ for all $i \in M$. $\quad V\left\{\mathfrak{A}_{i}: i \in I\right\}$ is the smallest variety containing all the $\mathfrak{A}_{i}$. If $\mathfrak{A}, \mathfrak{B}$ are varieties, $\mathfrak{A} \wedge \mathfrak{B}$ denotes their meet and $\mathfrak{A} \vee \mathfrak{B}$ denotes their join. If $A$ is an associative $\operatorname{ring}, \operatorname{var} A$ will denote the variety generated by $A$. Every variety $\mathscr{O}$ has free members of rank $1,2,3, \cdots$. The free member of $\operatorname{rank} n$ in $\mathscr{V}$ is denoted by $F_{n}(\mathscr{V})$. The varieties form a complete lattice $L$ with respect to $A$ and $V$. The least member of this lattice is the variety defined by $\{x=0\}$. The atoms of $L$ are the equationally complete varieties $[4,7]$ and they are given by $P(p, 1)$ and $Q(p)$ for all primes $p$, where $P(p, n)$ is the variety defined by $\left\{p x=0, x-x^{p^{n}}=0\right\}, Q(p)$ is the variety defined by $\{p x=0, x y=0\}$. Every variety contains an equationally complete variety.

The variety of all commutative rings is a first order definable member in the lattice of ring varieties. It is definable as the join of all immediate covers of 0 .

The following observation is useful.

LEMMA 1. The variety $\mathfrak{B}$ immediately covers the variety $\mathfrak{A}$ if and only if $\mathfrak{A}<\mathfrak{B}$ and every member of $\mathfrak{B}$ that does not belong to $\mathfrak{A}$ generates $\mathfrak{B}$.

This is clear since if $K<\mathfrak{B}, K$ cannot contain any member of $\mathfrak{B}$ outside $\mathfrak{A}$, and so $K \leqq \mathfrak{A}$. 
3. Now the immediate covers of some varieties will be determined.

THeOREM 2. A variety $\mathscr{C}$ is an immediate cover of $P(p, 1)$ if and only if $\mathscr{Q}=P(p, r)$, where $r$ is a prime.

Members $(\neq 0)$ of $P(p, r)$ are subdirect sums of subfields of the Galois field of $p^{r}$ elements. If $r$ is prime, the only subfields are $Z_{p}$, which generates $P(p, 1)$, and the Galois field of $p^{r}$ elements, which generates $P(p, r)$. Thus, if $r$ is prime, $P(p, r)$ is an immediate cover of $P(p, 1)$.

If $\mathscr{C}$ immediately covers $P(p, 1)$, it cannot contain any of the $Q(q)$ (by Lemma 1). Hence by a result of the author [3], $\mathscr{C}=$ $V\left\{P\left(p_{i}, r_{i}\right): 1 \leqq i \leqq k\right\}$. But $\mathscr{C}$ is join-irreducible, and so $\mathscr{U}=P\left(p_{1}, r_{1}\right)$, say. Also, $P(p, 1)<\mathscr{W}=P\left(p_{1}, r_{1}\right)$ implies that $p=p_{1}$. Unless $r_{1}$ is prime, $r_{1}$ has a nontrivial divisor $m$ and $P(p, 1)<P(p, m)<$ $P\left(p, r_{1}\right)=\mathscr{K}$.

Corollary 3. The variety $P(p)$ defined by $\{p x=0, x y-y x=0\}$ is the join of all immediate covers of $P(p, 1) . P(p)$ is first order definable in $L$ relative to $P(p, 1)$.

Every $P(p, 1)$ has an infinite number of immediate covers $P(p, r)$ with $\boldsymbol{r}$ prime. The ring $x \boldsymbol{Z}_{p}[x]$ generates $P(p) . \quad x \boldsymbol{Z}_{p}[x]$ is a subdirect sum of Galois fields of $p^{r}$ elements for infinitely many primes $r$.

4. In contrast to the varieties $P(p, 1)$, none of the varieties $Q(p)$ has more than three immediate covers.

THEOREM 4. The immediate covers of $Q(2)$ are the varieties defined by:

(i) $\{4 x=0, x y=0\}$, or

(ii) $\left\{2 x=0, x^{2}=0, x y z=0\right\}$.

The immediate covers of $Q(p), p \neq 2$ are the varieties defined by:

(1) $\left\{p^{2} x=0, x y=0\right\}$,

(2) $\{p x=0, x y-y x=0, x y z=0\}$, or

(3) $\left\{p x=0, x^{2}=0, x y z=0\right\}$.

Before proving Theorem 4, some of its applications will be shown.

It is immediate from Theorem 2.4 that 
Corollary 5. The variety defined by $\{2 x=0, x y=0\}$ is a first order definable member in the lattice of all varieties of associative rings.

It can be defined as the unique atom with precisely two immediate covers.

CoRollary 6. The variety defined by $\{x y=0\}$ is a first order definable member in the lattice of all varieties of associative rings.

It can be defined as the join of all atoms that have precisely three distinct immediate covers.

Corollary 7. The variety $P(2)$ defined by $\{2 x=0, x y-y x=0\}$ is a first order definable member in the lattice of all varieties of associative rings.

It can be defined as the variety that:

(1) contains $Q(2)$,

(2) is the join of all immediate covers of an atom with more than three immediate covers.

COROLLARY 8. The variety of all associative rings defined by $\{2 x=0\}$ is a first order definable member in the lattice of all associative ring varieties. It can be defined as the largest variety that meets the variety of all commutative rings in $P(2)$.

The following corollary will conclude the applications of Theorem 4.

Corollary 9. The variety of all Boolean rings $\left\{x-x^{2}=0\right\}$ is a first order definable member in the lattice of all varieties of associative rings.

It can be defined as the atom with more than three immediate covers contained in $P(2)$.

5. Proof of Theorem 4. First, all immediate covers of $Q(p)$ satisfy the identity $p^{2} x=0$.

Let $\mathscr{V}$ be an immediate cover of $Q(p)$. Consider $F_{1}(\mathscr{P})=A$ the free member of $\mathscr{Y}$ of rank $1 . A$ is generated by one element, say $a$. If the additive subgroup of $A$ generated by $a$ is infinite, then the ideal of $A$ generated by $\alpha^{2}$ is strictly contained in $A\left(\alpha \in\left(\alpha^{2}\right) \Rightarrow\right.$ $m a=0$ for some $m>0)$, and hence $A /\left(a^{2}\right)$ has nontrivial homomor- 
phic images satisfying $\{q x=0, x y=0\}$ for all primes $q$. That is, $\mathscr{V}$ contains all $Q(q)$ which contradicts the assumption that $\mathscr{V}$ immediately covers $Q(p)$. So $m a=0$ for some positive integer $m$, and $0<$ $s<m$ implies $s a \neq 0$. If $q$ is a prime divisor of $m$, then $q A=$ $\{q x: x \in A\}$ is an ideal of $A$; moreover if $q<m$, then $q A \neq A$, and $0 \neq B=A \mid q A \in \mathscr{Y}$. But $B$ satisfies $q x=0$. Unless $q=p, B \notin Q(p)$ and hence (by Lemma 1) $B$ generates $\mathscr{T}$, which is impossible since $Q(p)<\mathscr{V}$. Hence $p$ is the only prime divisor of $m$, i.e., $p^{\alpha} x=0$ is an identity in $\mathscr{T}$. If $\alpha \geqq 2$, i.e., $p x \neq 0$ in $\mathscr{Y}, A \mid p^{2} A \in \mathscr{V}$, $\operatorname{var}\left(A \mid p^{2} A\right) \leqq \mathscr{Y} ;$ and as $Q(p)<\operatorname{var}\left(A \mid p^{2} A\right), \quad \operatorname{var}\left(A \mid p^{2} A\right)=\mathscr{\mathscr { T }}$; i.e., $p^{2} x=0$ is an identity in $\mathscr{Y}$.

Also $x^{3}=0$ is an identity in $\mathscr{V}$. Let $C=A / p A$, where $A=$ $F_{1}(\mathscr{V}) . \quad C$ satisfies $p x=0$ and $C \in \mathscr{V} . \quad C \cong t \boldsymbol{Z}_{p}(t) \mid g(t) \boldsymbol{Z}_{p}[t]$, where $g(t)$ is a polynomial, with no absolute term, with coefficients from $Z_{p} . \quad g(t)=t^{r}+t^{r} g_{1}(t)$, where $g_{1}(t) \in t Z_{p}[t] . \quad$ As $C \neq 0, g(t) \neq t$ and $r \geqq 1$. Assume that $g_{1}(x) \neq 0$. Then the ideal $I=\left(t+t g_{1}(t)\right) Z_{p}[t]$ contains the ideal $g(t) \boldsymbol{Z}_{p}[t] . \quad t \boldsymbol{Z}_{p}[t] / I$ is a homomorphic image of $C$. Otherwise, since $t \boldsymbol{Z}_{p}[t] / I \neq 0, I=t \boldsymbol{Z}_{p}[t]$, and hence

$$
g(t)=t^{r-1}\left(t+t g_{1}(t)\right) \boldsymbol{Z}_{p}(t)=t^{r-1} I=t^{r} \boldsymbol{Z}_{p}[t],
$$

we would have $\boldsymbol{Z}_{p}$ as a homomorphic image of $t \boldsymbol{Z}_{p}[t] / I$ and hence $\boldsymbol{Z}_{p} \in \mathscr{Y}$, i.e., $\mathscr{V}$ contains $P(p, 1)$ which is impossible. Hence $C$ satisfies $x^{r}=0$. Hence, in $A, y^{r} \in p A$ for all $y \in A$, and thus $y^{2 r}=\left(y^{r}\right)^{2}=\left(p z^{2}\right)=$ $p^{2} z^{2}=0$, i.e., $y^{2 r}=0, r>0$, is an identity in $F_{1}(\mathscr{V})$ and hence also in $\mathscr{V}$. If $x^{2}=0$ is not an identity in $\mathscr{T}$, then $A /\left(\alpha^{3}\right)$ satisfies $x y z=0$, $x y \neq 0$ and hence generates the variety $\mathscr{Y}$, i.e., $\mathscr{C}$ satisfies $x^{3}=0$.

One now distinguishes between the cases where $p x=0$ is an identity in $\mathscr{T}$, and where $p x=0$ is not an identity in $\mathscr{V}$.

(a) $p^{2} x=0, p x \neq 0$.

In this case $0 \neq F_{1}(\mathscr{C}) / p F_{1}(\mathscr{V})$ does not generate $\mathscr{Y}$, and hence $\operatorname{var}\left(F_{1}(\mathscr{V}) / p F_{1}(\mathscr{Y})\right)=Q(p)$. Thus, the identity $x y=0$ is a consequence of the identities in $\mathscr{V}$ and $p x=0 ; x y=v(x, y)+p g(x, y)$, where $v(x, y)=0$ is an identity in $\mathscr{V}$. By putting one of the variables $x, y$ at a time equal to 0 , we can assume that both $v$ and $g$ are the sums of monomials involving both $x$ and $y$. Thus every monomial in $v$ and $g$ has $x y$ or $y x$ as a factor. Now

$$
p x y=p v(x, y)+p^{2} g(x, y) .
$$

As $p^{2} x=0$ is an identity in $\mathscr{V}$, we get that $p x y=0$ is an identity in $\mathscr{Y}$, and so $p g(x, y)=0$ is satisfied in $\mathscr{V}$. Hence $x y=v+$ $p g(x, y)=0$ is an identity in $\mathscr{T}$. Thus, if $\mathscr{Y}$ satisfies $p^{2} x=0$ but not $p x=0$, then $\mathscr{Y}$ satisfies $x y=0$. It is easy to check that the variety defined by $\left\{p^{2} x=0, x y=0\right\}$ is an immediate cover of $Q(p)$. 
(b) $\mathscr{V}$ satisfies $p x=0$. We have shown that $\mathscr{V}$ satisfies also $x^{3}=0$; we have two cases:

$\left(b_{1}\right) x^{2}=0$ is not an identity in $\mathscr{T}$.

Hence $F_{1}(\mathscr{V}) \cong x Z_{p}[x] / x^{3} Z_{p}[x] \in Q(p)$. Hence (by Lemma 1) $\mathscr{V}=$ $\operatorname{var} F_{1}(\mathscr{V})$, i.e., $\mathscr{V}$ is the variety defined by $\{p x=0, x y-y x=0$, $x y z=0\}$. This variety immediately covers $Q(p)$ if and only if $p \neq 2$.

Indeed any polynomial in one variable is equivalent (relative to $\mathscr{V})$ to a polynomial of the type:

$$
\alpha x+\beta x^{2} \text {. }
$$

If $\alpha \neq 0$, such a polynomial identity is equivalent to $x=-\gamma x^{2}$ implying $x=\gamma^{2} x^{3}=0$. If $\alpha=0$ and $\beta \neq 0$ it is equivalent to $x^{2}=0$, implying $x y+y x=0$. As $p \neq 2$, this implies $x y=0$ (relative to $\mathscr{V}$ ).

Any polynomial in two variables is equivalent (relative to $\mathscr{V}$ ) to a polynomial of the type

$$
f(x)+g(y)+\alpha x y
$$

where $f$ and $g$ are polynomials in one variable. Thus any variety satisfying the identities of $\mathscr{V}$ and a polynomial of this type will satisfy $f(x)=0=g(y)$ and $\alpha x y=0$. If this polynomial is nontrivial, one can derive $x=0$ or $x y=0$ from it (relative to $\mathscr{V}$ ). The same is true for polynomial identities in more than two variables

$\left(b_{2}\right) x^{2}=0$ is an identity in $\mathscr{V}$

$$
F_{1}(\mathscr{V})=x Z_{p}[x] / x^{2} Z_{p}[x] . \quad Q(p)=\operatorname{var}\left(F_{1}(\mathscr{V})\right) .
$$

Since $\mathscr{V}$ satisfies $x^{2}=0$, it satisfies also $x y+y x=0$. The elements of $F_{2}(\mathscr{V})$ can be written in the form

$$
\alpha x+\beta y+\gamma x y+\delta y x
$$

because any monomial of degree $>2$ involves (because $x y=-y x$ ) $x^{2}$ or $y^{2}$ and hence is 0 .

In $F_{2}(\mathscr{Y})$ the product of any three elements is zero but $x y \neq 0$ in $F_{2}(\mathscr{V})$. Thus $F_{2}(\mathscr{V}) \notin Q(p)$ and again by Lemma $1 \operatorname{var}\left(F_{2}(\mathscr{V})\right)=\mathscr{V}$, i.e., $\mathscr{V}$ satisfies also $x y z=0 . \quad \mathscr{V} \equiv\left\{p x=0, x^{2}=0, x y z=0\right\} \quad \mathrm{im}$ mediately covers $Q(p)$ for all $p$, including $p \equiv 2$.

As $x^{2}=0$ in $\mathscr{T}$, the nontrivial polynomials in one variable would lead to $x=0$ (in $\mathscr{V}$ ). Nontrivial polynomials of two variables will lead in $\mathscr{V}$ to $\gamma x y+\delta y x$. An identity $\gamma x y+\delta y x=0$ is equivalent (relative to $\mathscr{P}$ ) to one of the identities $x y=0, x y-y x=0, x y+$ $y x=0$. The third one is trivial, the second is equivalent to the first if $p \neq 2$, and to the third if $p=2$. The first implies that the new variety is contained in $Q(p)$. 
In case $p=2$, the variety $\{2 x=0, x y-y x=0, x y z=0\}$ strictly contains the variety $\left\{2 x=0, x^{2}=0, x y z=0\right\}$, and cannot immediately cover $Q(2)$.

In conclusion it should be noticed that considering only commutative rings Theorem 4 would not distinguish between $Q(2)$ and $Q(p)$, $p \neq 2$. Thus the results of this paper cannot be immediately applied to the lattice of varieties of commutative rings.

The author is grateful to the referees for a number of comments.

\section{REFERENCES}

1. P. M. Cohn, Universal Algebra, Harper \& Row, New York, 1965.

2. G. Grätzer, Universal Algebra, Van Nostrand, Princeton, 1968.

3. A. A. Iskander, Product of ring varieties and attainability, Trans. Amer. Math. Soc., 193 (1974), 231-238.

4. J. Kalicki and D. Scott, Equational completeness of abstract algebras, Indag. Math., 17 (1955), 650-659.

5. R. McKenzie, Definability in lattices of equational theories, Annals Math. Logic, 3: 2 (1971), 197-237.

6. J. M. Osborn, Varieties of algebras, Advances in Math., 8: 2 (1972), 163-369.

7. A. Tarski, Equationally complete rings and relation algebras, Indag. Math., 18 (1956), 39-46.

Received June 22, 1973 and in revised form October 3, 1977.

UNIVERSITY OF SOUTHWESTERN LOUISIANA

LAFAYETTE, LA 70501

AND

KUWAIT UNIVERSITY

KUWAIT, KUWAIT 



\section{PACIFIC JOURNAL OF MATHEMATICS}

\section{EDITORS}

RICHARD ARENS (Managing Editor)

University of California

Los Angeles, California 90024

C. W. CURTIS

University of Oregon

Eugene, OR 97403

C. C. MOORE

University of California

Berkeley, CA 94720

\section{J. DUGUNDJI}

Department of Mathematics University of Southern California Los Angeles, California 90007

R. Finn aNd J. Milgram Stanford University Stanford, California 94305

\section{ASSOCIATE EDITORS}

E. F. BeCKenbaCH

B. H. NeumanN

F. WOLF

K. YosHIDA

\section{SUPPORTING INSTITUTIONS}

UNIVERSITY OF BRITISH COLUMBIA CALIFORNIA INSTITUTE OF TECHNOLOGY UNIVERSITY OF CALIFORNIA MONTANA STATE UNIVERSITY UNIVERSITY OF NEVADA, RENO NEW MEXICO STATE UNIVERSITY OREGON STATE UNIVERSITY UNIVERSITY OF OREGON
UNIVERSITY OF SOUTHERN CALIFORNIA STANFORD UNIVERSITY UNIVERSITY OF HAWAII UNIVERSITY OF TOKYO UNIVERSITY OF UTAH WASHINGTON STATE UNIVERSITY UNIVERSITY OF WASHINGTON 


\section{Pacific Journal of Mathematics}

\section{Vol. 76, No. $1 \quad$ November, 1978}

Ata Nuri Al-Hussaini, Potential operators and equimeasurability ......... 1

Tim Anderson and Erwin Kleinfeld, Semisimple nil algebras of type $\delta . \ldots .99$

Stephen LaVern Campbell, Linear operators for which $T^{*} T$ and $T+T^{*}$

commute. III ......................................

Robert Jay Daverman, Special approximations to embeddings of codimension one spheres...............................

Donald M. Davis, Connective coverings of $\mathrm{BO}$ and immersions of projective

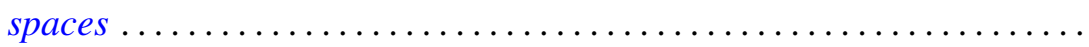

V. L. (Vagn Lundsgaard) Hansen, The homotopy type of the space of maps of

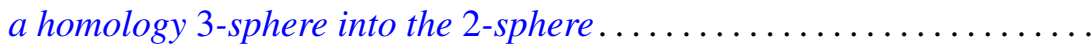

James Victor Herod, A product integral representation for the generalized

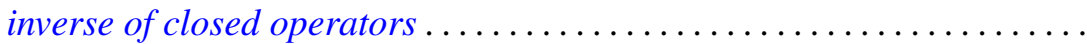

A. A. Iskander, Definability in the lattice of ring varieties ..............

Russell Allan Johnson, Existence of a strong lifting commuting with a

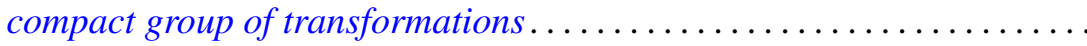

Heikki J. K. Junnila, Neighbornets...................... 83

Klaus Kalb, On the expansion in joint generalized eigenvectors . ......... 109

F. J. Martinelli, Construction of generalized normal numbers . . . . . . . . . 117

Edward O'Neill, On Massey products ....................... 123

Vern Ival Paulsen, Continuous canonical forms for matrices under unitary

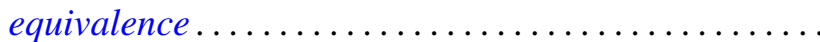

Justin Peters and Terje Sund, Automorphisms of locally compact groups . . . 143

Duane Randall, Tangent frame fields on spin manifolds . . . .

Jeffrey Brian Remmel, Realizing partial orderings by classes of co-simple sets . . . .

J. Hyam Rubinstein, One-sided Heegaard splittings of 3-manifolds ...

Donald Charles Rung, Meier type theorems for general boundary approach

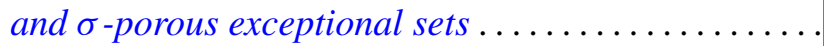

Ryōtarō Satō, Positive operators and the ergodic theorem

Ira H. Shavel, A class of algebraic surfaces of general type constructed from

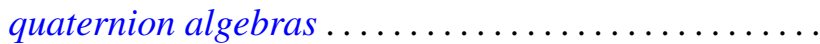

Patrick F. Smith, Decomposing modules into projectives and injectives ....

Sergio Eduardo Zarantonello, The sheaf of outer functions in the polydisc... 